\title{
Wird die delegierte Psychotherapie abgeschafft?
}

\author{
Dr. med. J. de Haller, Allgemeinmedizin FMH, Präsident der SGAM
}

Dr. med. P. Loeb, Allgemeine Medizin, Präsident APPM

Dr. med. Ch. Bernath, FMH Psychiatrie und Psychotherapie, Co-Präsident IGDP

Die Ärztliche Interessengemeinschaft für Delegierte Psychotherapie (IGDP) hat der Kommission für Weiter- und Fortbildung (KWFB) den Antrag gestellt, auf eidgenössischer Ebene einen Fähigkeitsausweis für die delegierte Psychotherapie zu schaffen. Dieser sah vor, dass die Inhaber des neuen FA Delegierte Psychotherapie, die Titelträger des FA für Psychosomatische und Psychosoziale Medizin sowie die FachärztInnen für Psychiatrie und Psychotherapie sowie Kinder- und Jugendpsychiatrie und -psychotherapie delegieren dürfen.

Die KWFB hat diesen Antrag aus mehreren Gründen und unterschiedlichen Zielen abgelehnt. Die SGAM vor allem deswegen, weil es sich bei der delegierten Psychotherapie um ein Relikt aus alten Zeiten handelt, das - unter annehmbaren Übergangsregelungen - unbedingt verlassen werden muss, und das als nichtärztliche Leistung in einem modernen Tarif wie dem TARMED nichts mehr zu suchen hat. Die nichtärztliche Psychotherapie, die unbestritten durchaus ihre wertvollen Dienste leistet, bedarf - wie zum Beispiel die Physiotherapie - einer eigenen Tarifierung, die mit den entsprechenden Instanzen separat geregelt werden muss. Die Psychiater haben den Antrag vor allem deswegen verworfen, weil sie wesentlich höhere Anforderungen an die delegierenden Ärzte/-innen stellen möchten, nämlich eine vollständige Psychotherapieausbildung.

Von Gesetzes wegen ist es heute - vor der Einführung von TARMED - allen Ärzten/-innen erlaubt, Psychotherapie zu delegieren. Die Krankenversicherer bestehen aber teilweise jetzt schon auf ihrer Pflicht und ihrem Recht, qualitative Anforderungen zu stellen. So halten beispielsweise im Kanton Zürich die IGDP zusammen mit der santésuisse Zürich-Schaffhausen Unbedenklichkeitskriterien sowohl für die delegierenden Ärzte/-innen als auch für die angestellten nichtärztlichen Psychotherapeuten/-innen erarbeitet. Dies kann grundsätzlich als sinnvoll erachtet und, wo erwünscht, auf kantonaler Ebene durchgeführt werden.
Aus der Summe der erwähnten übergeordneten Gründe hat sich die KWFB fast ohne Gegenstimmen trotzdem klar gegen eine solche Regelung auf eidgenössischer Ebene mittels eines Fähigkeitsausweises ausgesprochen. Die delegierte Psychotherapie soll aus dem TARMED gekippt werden und bis dahin im Sinne einer einfachen und gerechten Übergangsregelung weiterhin allen Ärztinnen offenstehen.

Der FMH-Präsident will nun gemäss seinem Schreiben vom 28.10.2002 den Zulassungspassus im TARMED 1.1. trotzdem so belassen, wie er ist. Das heisst, dass mit Beginn des TARMED nur noch die Fachärzte/-innen für Psychiatrie und Psychotherapie sowie Kinder- und Jugendpsychiatrie und -psychotherapie delegieren dürfen. Das entspricht keineswegs dem, was die KWFB-Delegierten mit ihrem Entscheid bewirken wollten.

Eine derartige Einschränkung einer ärztlichen Tätigkeit durch neue Dignitätsanforderungen, welche sich die bisherigen Anbieter in einer vertretbaren Übergangszeit nicht aneignen können, ist auch gemäss gültigem Recht nicht erlaubt und kann jederzeit angefochten werden.

Es ist allen klar, dass die delegierte Psychotherapie als Konstrukt verschwinden wird, sobald die nichtärztliche selbständige Psychotherapie zu Lasten der Grundversicherung angeboten werden kann. Die jetzige Formulierung im TARMED würde das «Aus» für viele Psychotherapien in nichtpsychiatrischen Praxen bedeuten. Bis es aber soweit ist, müssen wir eine für die Ärzte/-innen und Patienten/-innen sowie die nichtärztlichen Psychotherapeuten/-innen gangbare Übergangsregelungen finden.

Die Ärztekammer und die Präsidentenkonferenz müssen also das Geschäft dringend wieder aufnehmen und einen realistischen Entscheid fällen. 